\title{
Evaluación de la aclimatación y rendimiento de 20 cultivares de brócoli (Brassica oleracea L. Var. Itálica.), a campo abierto
}

\author{
Evaluation of acclimatization and yield of 20 broccoli cultivars (Brassica oleracea L. Var. \\ Itálica.), in the open field \\ Aclimatação e avaliação do rendimento de 20 cultivares de brócolis (Brassica oleracea L. \\ Var. Itálica.), Em campo aberto \\ Cristian M. Parra Morocho \\ kristianp_117@yahoo.es \\ Luis Hidalgo \\ hidalgo.f20@yahoo.com
}

\begin{abstract}
Facultad de Recursos Naturales de la Escuela Superior Politécnica de Chimborazo, Ecuador
\end{abstract}
Artículo recibido enero 2018, arbitrado febrero 2018 y publicado en mayo 2018

\section{RESUMEN}

El presente artículo muestra la evaluación de la aclimatación y rendimiento de 20 cultivares de brócoli (Brassica oleracea L. Var Itálica.), en Guayllabamba, Cantón Chambo, Provincia de Chimborazo; la metodología utilizada fue ADEVA del diseño de Bloques Completos al Azar (BCA), estableciendo parcelas con 20 cultivares de brócoli y tres repeticiones. Los cultivares que mejor se aclimataron a las condiciones de Guayllabamba fueron Interoc 010 (T17), Interoc 006 (T14), Hot Bro (T8), y Avenger (T19) por sus características morfológicas y fisiológicas.; Interoc 010 (T17) e Interoc 006 (T14) presentaron la mayor altura de planta con $91,18 \mathrm{~cm}$ y $85,74 \mathrm{~cm}$ respectivamente, el mayor número de hojas presentaron los cultivares Interoc 001 (T11) e Interoc 006 (T14) con 17,67 y 17,63 hojas; para número de días a inicio de la cosecha Hot Bro (T8) fue el cultivar más precoz con 78 días y Steel (T18) se consideró como un cultivar tardío con 84,33 días; los mayores diámetros de pella presentaron los cultivares Legacy (T20) e Interoc 001 (T11) con 18,08 y 17,87 cm respectivamente; las mejores características de la pella (forma, compactación, granulometría y color) presentaron los cultivares 25-455 (T2), Interoc 010 (T17), Avenger (T19) y Legacy (T20); los mayores rendimientos en campo por ha presentaron los cultivares Hot Bro (T8) e Interoc 010 (T17), con 37390 $\mathrm{kg}$ y $37220 \mathrm{~kg}$ respectivamente y en agroindustria los cultivares Interoc 010 (T17), Avenger (T19) con 25870 $\mathrm{kg}$ y $25390 \mathrm{~kg}$ respectivamente; para el rendimiento industrial procesado los mejores cultivares fueron Interoc 009 (T16) e Interoc 001 (T11) con 75,24 \% y $74,37 \%$ respectivamente; económicamente el cultivar Interoc 010 (T17) presentó el mayor beneficio neto por hectárea con 4353, 89 USD y una tasa de retorno marginal de 1513,94\%.

Palabras clave: Brassica oleracea L. Var. Itálica; aclimatación y rendimiento; cultivos de brócoli

\begin{abstract}
This article shows the evaluation of acclimatization and yield of 20 broccoli cultivars (Brassica oleracea L. Var Itálica.), In Guayllabamba, Canton Chambo, Province of Chimborazo; The methodology used was ADEVA of the design of Random Complete Blocks (BCA), establishing plots with 20 broccoli cultivars and three repetitions. The cultivars that best acclimated to the conditions of Guayllabamba were Interoc 010 (T17), Interoc 006 (T14), Hot Bro (T8), and Avenger (T19) for their morphological and physiological characteristics . Interoc 010 (T17) and Interoc 006 (T14) had the highest plant height with $91.18 \mathrm{~cm}$ and $85.74 \mathrm{~cm}$ respectively, the largest number of leaves presented the Interoc 001 (T11) and Interoc 006 (T14) cultivars with 17,67 and 17.63 sheets; for number of days at the beginning of the harvest Hot Bro (T8) was the earliest cultivar with 78 days and Steel (T18) was considered as a late cultivar with 84.33 days; the largest pellet diameters presented the Legacy (T20) and Interoc 001 (T11) cultivars with 18.08 and 17.87 $\mathrm{cm}$ respectively; the best characteristics of the pellet (shape, compaction, grain size and color) presented cultivars 25-455 (T2), Interoc 010 (T17), Avenger (T19) and Legacy (T20); the highest yields in the field per ha presented the Hot Bro (T8) and Interoc 010 (T17) cultivars, with $37390 \mathrm{~kg}$ and $37220 \mathrm{~kg}$ respectively and in the agro-industry the Interoc 010 (T17), Avenger (T19) cultivars with $25870 \mathrm{~kg}$ and $25390 \mathrm{~kg}$ respectively; for the processed industrial yield the best cultivars were Interoc 009 (T16) and Interoc 001 (T11) with $75.24 \%$ and $74.37 \%$ respectively; Economically, cultivating Interoc 010 (T17) presented the highest net profit per hectare with 4353,89 USD and a marginal return rate of $1513.94 \%$.
\end{abstract}

Key words: Brassica oleracea L. Var. Italic; acclimatization and performance; broccoli crops 


\section{RESUMO}

Este artigo mostra a avaliação da aclimatação e do rendimento de 20 cultivares de brócolis (Brassica oleracea L. Var Itálica.), Em Guayllabamba, Cantão de Chambo, província de Chimborazo; A metodologia utilizada foi a ADEVA, no delineamento de Blocos Aleatórios Completos (BCA), estabelecendo parcelas com 20 cultivares de brócolis e três repetições. As cultivares que melhor se acostumaram às condições de Guayllabamba foram Interoc 010 (T17), Interoc 006 (T14), Hot Bro (T8) e Avenger (T19) por suas características morfológicas e fisiológicas. Interoc 010 (T17) e Interoc 006 (T14) apresentaram a maior altura de planta com $91,18 \mathrm{~cm}$ e $85,74 \mathrm{~cm}$, respectivamente, o maior número de folhas apresentou as cultivares Interoc 001 (T11) e Interoc 006 (T14) com 17, 67 e 17,63 folhas; por número de dias no início da colheita, o Hot Bro (T8) foi a cultivar mais precoce com 78 dias e o Steel (T18) foi considerado como cultivar tardia com 84,33 dias; os maiores diâmetros de pellets apresentaram as cultivares Legacy (T20) e Interoc 001 (T11) com 18,08 e 17,87 cm, respectivamente; as melhores características do granulado (forma, compactação, tamanho e cor dos grãos) apresentaram as cultivares 25-455 (T2), Interoc 010 (T17), Avenger (T19) e Legacy (T20); os maiores rendimentos no campo por ha apresentaram as cultivares Hot Bro (T8) e Interoc 010 (T17), com $37390 \mathrm{~kg}$ e $37220 \mathrm{~kg}$ respectivamente e na agroindústria as cultivares Interoc 010 (T17), Avenger (T19) com $25870 \mathrm{~kg}$ e $25390 \mathrm{~kg}$ respectivamente; para o rendimento industrial processado, as melhores cultivares foram Interoc 009 (T16) e Interoc 001 (T11) com 75,24\% e 74,37\%, respectivamente; Economicamente, o cultivo do Interoc 010 (T17) apresentou o maior lucro líquido por hectare, com 4353, 89 USD e uma taxa de retorno marginal de $1513,94 \%$

Palavras-chave: Brassica oleracea L. Var. Itálico; aclimatação e desempenho; culturas de brócolis

\section{INTRODUCCIÓN}

Ecuador es un país que no posee las cuatro estaciones climáticas, no dispone de las condiciones ambientales adecuadas para la producción de semillas de brócoli (Brassica oleracea. L. Var. Itálica), por tal motivo, las casas comerciales importan el material genético desde otros países de cuatro estaciones, para ser comercializadas sin realizar un previo ensayo en las zonas de producción y determinar su capacidad de aclimatarse o no a las condiciones de las zonas de producción.
El crecimiento del cultivo comercial de brócoli en Ecuador se inició en 1990, cuando crecientes superficies de terreno se destinaron a este producto; los productores hablan de un crecimiento imparable de este producto de exportación no tradicional, que se asomó en el mercado nacional hace una década. Desde entonces se han cultivado entre 3000 y 5000 hectáreas en la Sierra: de Cotopaxi sale la mayor producción (68\%); luego le sigue Pichincha, con el 16\%; Imbabura, 10\%; Carchi, 3\%; Chimborazo, 2\%; y el resto del país, 1\%. (Encarta, 2007).

La evaluación hace referencia a un proceso por medio del cual alguna o varias características de un grupo de materiales o tratamientos, programas, etc., reciben la atención de quien evalúa, se analizan y se valoran sus características y condiciones en función de parámetros de referencia para emitir un juicio que sea relevante para el evaluador (Tyler, 1973).

La aclimatación hace referencia a aquellas modificaciones heredables $\mathrm{y}$, por tanto, incluidas en la información genética, que se manifiesta de forma transitoria en la fisiología de la planta, para aumentar la probabilidad de que la misma sobreviva y se reproduzca en un ambiente particular y, por tanto es reversible. (Azcón, y Talón, 2000).

Rendimiento agronómico es planteado como la eficacia de todos los recursos utilizados en un proceso productivo, para lograr los objetivos agronómicos planteados, incluyendo la reducción de riesgos y mejorando la calidad de las cosechas (Fraume, 2007).

Además, cultivar es planteado como la variedad de cualquier especie vegetal cultivada en contraposición con aquélla que crece en estado silvestre. El término es una contracción de las palabras 'variedad cultivada' y suele abreviarse como cv. (Encarta 2007). 
El brócoli pertenece al orden Rhoedales,

RESULTADOS familia Brassicaceae, su nombre científico es Brassica oleraceae, Var Itálica, es una hortaliza con un desarrollo floral rápido y es originario del Mediterráneo y Asia Menor. Su aporte de vitamina C, B2 y vitamina A es elevado; además suministra cantidades significativas de minerales. La planta de brócoli forma un tipo de cabeza que consiste en unos brotes verdes y los tallos de la flor carnosos y espesos los cuales son más largos que los de la coliflor, la raíz es pivotante, las hojas son algo rizadas y de color verde oscuro, las flores del brócoli son pequeñas, en forma de cruz de color amarillo, las inflorescencias están constituidas por primordios foliares, también llamados flores inmaduras dispuestas en un corimbo primario en el extremo superior del tallo, los corimbos son de color variado según el cultivar de verde claro o verde púrpura, el fruto es una silicua de valvas ligeramente convexas con un solo nervio longitudinal, el brócoli produce abundantes semillas redondas y de color rosáceo. (Pantoja, 2014).

\section{MATERIALES Y MÉTODOS}

El estudio de llevo a cabo en Guayllabamba, Cantón Chambo, Provincia de Chimborazo. La investigación fue de tipo experimental. La distancia de trasplante: Entre plantas fue de 0,30 m; entre hileras de 0,60 m, el área total del ensayo fue de $850 \mathrm{~m}^{2}$, el área total de la parcela de $9(3 * 3) \mathrm{m}^{2}$; número de plantas/parcela fue de $50(10 * 5)$; y por último estufo conformada también con número de plantas evaluadas de 10. Se utilizó el diseño de Bloques Completos al Azar (BCA), con 20 tratamientos y tres repeticiones. Para el análisis funcional se determinó el coeficiente de variación, en porcentajes, se realizó la prueba de Tukey al 5\% además de un análisis económico según Perrin.

\section{Porcentaje de germinación}

Presentaron un 100\% de germinación los cultivares; 25-571 (T1), Elsinore (T4), Interoc 008 (T15) e Interoc 009 (T16) y el cultivar Interoc 001 (T11) obtuvo el más bajo porcentaje de germinación con un $90 \%$

\section{Porcentaje de emergencia}

El cultivar que obtuvo el $100 \%$ de emergencia es el 25-571 (T1), mientras que el cultivar que presentó un porcentaje bajo de emergencia fue el cultivar Interoc 005 (T13) con un $86 \%$.

\section{Porcentaje de prendimiento}

El cultivar que alcanzó mayor porcentaje de prendimiento fue el Interoc 010 (T17) con 99,33\%. Mientras que el cultivar que presentó menor porcentaje de prendimiento fue Interoc 005 (T13), con 93,33\%.

\section{Altura de la planta}

A los 15 días después del trasplante, los cultivares que presentaron mayor altura fueron Hot Bro (T8) e Interoc 010 (17), con medias de 8,61 y 8,39 cm respectivamente, mientras que el cultivar que obtuvo menor altura fue Interoc 005 (T13) con $7 \mathrm{~cm}$. Sin embargo a los 30 días después del trasplante, el cultivar que presentó mayor altura fue Hot Bro (T8) con una media de 22,75 cm respectivamente, mientras que el cultivar Interoc 005 (T13) fue el que presentó menor altura con una media de 17,96 cm. respectivamente. En cambio a los 45 días después del trasplante, los cultivares que alcanzaron mayor altura fueron Hot Bro (T8) y Tahoe (T3) con medias de 46,75 y 46,13 cm respectivamente y finalmente a los 60 días después del trasplante, el cultivar que alcanzó mayor altura fue Interoc 010 (T17) con una 
media de 91,18 cm, superando a los cultivares comerciales Avenger (T19) y Legacy (T20) que alcanzaron alturas de 83,16 y 74,81 cm respectivamente; los cuales a su vez superaron al cultivar Verdia (T9), que al final de la fase de crecimiento presentó una altura menor con respecto a los demás cultivares con una media de $64,13 \mathrm{~cm}$.

\section{Número de hojas}

El cultivar que presentó mayor número de hojas a los 60 días, fue Interoc 001 (T11) con una media de 17,67 superando a los cultivares comerciales Avenger (T19) y Legacy (T20) que presentaron medias de 15,80 y 15,87 respectivamente, mientras que el cultivar Steel (T18) fue el que presentó menor número de hojas, con una media de 12,10

\section{Número de brotes laterales por planta}

El cultivar que presentó mayor número de brotes laterales fue Elsinore (T4) con una media de 4,40; superando a los cultivares comerciales Avenger (T19) y Legacy (T20) que presentaron medias de 1,13 y 1,20 respectivamente,

\section{Número de días a la aparición de la pella}

El cultivar que obtuvo menor número de días a la aparición de pella fue Hot Bro (T8) con una media de 58,30 días, mientras que el cultivar Steel (T18) con una media de 64,30 días fue el que mayor número de días obtuvo a la aparición de la pella

\section{Días a inicio de la cosecha y precocidad}

Steel (T18) con 84,33 días se interpretó como tardío, mientras Hot Bro (T8) con 78 días se interpretó como un cultivar "medio".

\section{Características de la pella}

\section{Diámetro ecuatorial de la pella}

Legacy (T20) alcanzó el mayor diámetro de pella con $18,08 \mathrm{~cm}$

\section{Compactación de la pella}

Todos los cultivares se interpretaron como pellas compactas a excepción del cultivar Interoc 008 (T15) que obtuvo una pella ligeramente compacta.

\section{Granulometría}

24-455 (T2), Elsinore (T4) e Interoc 008 (T15) presentaron una granulometría gruesa.

\section{Color de la pella}

Los cultivares que mostraron una coloración verde azulado fueron King Dome (T7), Hot Bro (T8), Verdia (T9), Interoc 003 (T12), Interoc 008 (T15), Interoc 010 (T17), Steel (T18) y Legacy (T20)

\section{Forma de la pella}

Los cultivares 24-455 (T2), Interoc 008 (T15) e Interoc 010 (T17) mostraron una forma de pella piramidal.

\section{Peso de la pella (gramos)}

Los cultivares Hot Bro (T8) e Interoc 010 (T17), que alcanzaron mayor peso de pella, con medias de 673 y 670 g respectivamente, y el cultivar Interoc 009 (T16), que presentó menor peso de pella, con una media de 526,30 g.

\section{Rendimiento en campo y agroindustria en $\mathrm{kg} / \mathrm{ha}$}

En campo Hot Bro (T8) e Interoc 010 (T17) obtuvieron mayor rendimiento por hectárea, con 37390 y $37220 \quad \mathrm{~kg}$ respectivamente. En agroindustria Interoc 
010 (T17) y Avenger (T19) presentaron el mayor rendimiento/ ha con 25870 y 25390 $\mathrm{kg}$ respectivamente.

\section{Porcentaje de rendimiento industrial procesado}

Interoc 009 (T16) presentó mayor porcentaje de rendimiento industrial procesado, con medias de $75,24 \%$.

\section{Análisis económico}

Legacy (T20) presentó el mayor costo variable por hectárea con 420,00 USD; Interoc 010 (T17) mostró el mayor beneficio neto con 4353,89 USD; los tratamientos no dominados fueron Verdia (T9) e Interoc 010 (T7), la mayor tasa de retorno marginal presentó Interoc 010 (T17) con 1513,94\%.

\section{CONCLUSIONES}

Los cultivares que mejor se aclimataron a las condiciones ambientales de Guayllabamba, Cantón Chambo, Provincia de Chimborazo fueron Interoc 010 (T17), Interoc 006 (T14), Hot Bro (T8), y Avenger (T19); que sobresalieron por sus características fisiológicas y morfológicas como altura, número de hojas, número de brotes laterales, precocidad, características de la pella (diámetro, color, compactación, forma, granulometría y peso). Sin embargo el cultivar Hot Bro (T8) presentó uno de los mayores pesos de pella.
Utilizar desde el punto de vista de la aclimatación y económico en Guayllabamba, Cantón Chambo, Provincia de Chimborazo los cultivares Interoc 010 (T17), Avenger (T19), Interoc 006 (T13) y Hot Bro (T8); por sus excelentes características fisiológicas, morfológicas y económicas presentadas en esta investigación.

Realizar investigaciones con los cultivares Hot Bro (T8) e Interoc 010 (T17) debido a sus mayores rendimientos por hectárea de $37390 \quad \mathrm{~kg} \quad \mathrm{y} \quad 37220 \quad \mathrm{~kg}$ respectivamente.

\section{REFERENCIAS}

Azcón, J. Y Talón, M. (2000). “Fundamentos de la Fisiología Vegetal" 1ra ed. McGraw-WillInteramericana. Barcelona (España). p.481

Encarta ${ }^{\circledR}$ (2007). (C) 1993-2006 Microsoft Corporation. Reservados todos los derechos

Fraume, N. (2007). "Diccionario ambiental". Editorial Kimpres Ltda. Bogotá Colombia. P. 465

Pantoja Gordón, R. F. (2014). Evaluación de diferentes dosis de abonos orgánicos de origen animal en el comportamiento agronómico, del cultivo de brócoli en la zona de Huaca, Provincia del Carchi (Bachelor's thesis, Babahoyo: UTB, 2014)

Tyler, R. (1973). Introducción Cap. 1. Principios básicos del currículo 